\title{
Effect of Germinated Maize Based Diets on Biochemical Parameters in Kadaknath
}

\author{
Akhelesh Kumar Karoriya, Archana Jain*, M. K. Mehta, Chouhan, \\ Laxmi, Kumar, Rakesh and J. Shakkarpude \\ Department of Veterinary Physiology and Biochemistry, College of Veterinary Science and \\ A.H., Mhow, Nanaji Deshmukh Veterinary Science University (M.P.), India \\ *Corresponding author
}

\section{A B S T R A C T}

\begin{tabular}{|l|}
\hline Ke y w o r d s \\
$\begin{array}{l}\text { Poultry farming, } \\
\text { chicken, turkeys, } \\
\text { ducks etc }\end{array}$ \\
\hline Article Info \\
\hline $\begin{array}{l}\text { Accepted: } \\
\text { 12 August } 2019 \\
\text { Available Online: } \\
\text { 10 September } 2019\end{array}$ \\
\hline
\end{tabular}

\section{Introduction}

Poultry farming is the practice of raising poultry, chicken, turkeys, ducks etc. as an important subcategory of animal husbandry for the production of eggs, meat, feather, etc. Backyard poultry keeping is practiced by majority of the poor and marginalized rural households all over India. These households rely on low cost backyard poultry rearing to supplement and enhance their livelihoods.

Kadaknath is an important Indian poultry breed, also known as Kalamashi in Hindi, is 
known for its black-colored meat, poor egg production, slow growth rate, smaller body size as well as late sexual maturity. The commonly available colours of Kadaknath are jet-black, pencilled and golden. In all the three varieties of Kadaknath breed, most of the internal organs exhibit intense black coloration which is due to the deposition of melanin pigment in the connective tissue of organs and in the dermis (Rao and Thomas 1984). It is being reared by tribal communities in its breeding tract of the Jhabua and Dhar districts in the western region of the state of Madhya Pradesh and in adjoining areas of the states of Gujarat and Rajasthan. Although the meat of this breed has an unattractive appearance, it has a delicious flavor (Panda and Mahapatra, 1989). The flesh of this breed is black, it is considered not only for the delicacy of distinctive taste and flavor, but also of medicinal value.

Maize is one of the main cereals used worldwide in poultry ration. It is free of viscous non-starch polysaccharides, the major anti-nutritive factor present in most other cereals like barley, wheat and oats. At present the feed industry is using maize as a basic energy source in poultry diets. The nutritive value of maize seed depends on the technological processes used, such as milling, oil extraction, starch separation and germination.

The nutritive value of maize and other cereals depends on germination. One of the most important methods to increase the nutritive value of the grains, cereal and oil seeds is to germinate them. The nutritional value of sprouted grains is improved due to the conversion of complex compounds into relatively simpler compounds that are nutritionally more valuable. Sprouting of grains has resulted in increased protein quantity and quality. Sprouting also increases the concentration of certain nutrients including sugars, minerals and vitamin contents. However, sprouting has resulted in decreased starch contents and dry matter content of grains. It also increases the enzyme contents of grains.

\section{Materials and Methods}

The present experiment was conducted on 128 day old Kadaknath chicks belonging to same hatch and randomly divided into four treatment groups of 32 chicks each viz., T0, T1, T2 and T3. Each group was further divided into four replications of 08 birds each. The duration of the experiment was carried out for 240 days. Control groups (T0) was given standard ration with $100 \%$ normal maize based diet. The treatment Group T1 was given diet containing standard ration with $50 \%$ germinated maize.

The Group T2 was given diet containing standard ration with $75 \%$ germinated maize and Group T3 was given diet containing 100\% germinated Maize. The experimental birds were vaccinated against Marek's disease, Ranikhet disease and Gumboro disease/IBD at appropriate time. The birds were housed in deep litter system in the well ventilated and lighted rooms and the feed was given $a d . l i b$. with plenty of fresh water. The body weight of day old chicks was recorded on arrival and thereafter on weekly intervals.

Blood was collected on every 30th days from each bird. About 3-4ml was collected from wing vein using 22-24 gauge needle in a clean dry vial for biochemical analysis. The blood samples were centrifuged at $3000 \mathrm{rpm}$ for 10 minutes at room temperature and separated serum stored at $4^{\circ} \mathrm{C}$ till further analysis. The biochemical study was carried out using ERBA standard diagnostic kits. All observations were analyzed by using ANOVA (Snedecor and Cochran, 1994) to compare different treatment group. 


\section{Results and Discussion}

\section{Calcium and phosphorus}

The mean values of Calcium for T0, T1, T2 and T3 were $10.06 \pm 0.01,11.36 \pm 0.01$, $12.36 \pm 0.02$ and $11.03 \pm 0.05$, after 30 days, $10.72 \pm 0.05, \quad 11.63 \pm 0.07, \quad 12.44 \pm 0.05$ and $11.31 \pm 0.03$, after 60 days, $10.97 \pm 0.02$, $11.93 \pm 0.02,12.74 \pm 0.01$ and $11.66 \pm 0.01$, after 90 days, $10.33 \pm 0.01,12.03 \pm 0.04,12.96 \pm 0.01$, and $11.88 \pm 0.02$, after 120 days, $10.32 \pm 0.01$, $12.15 \pm 0.10,13.09 \pm 0.02$ and $12.12 \pm 0.01$, after 150 days, $10.63 \pm 0.01,12.32 \pm 0.05,13.34 \pm 0.01$ and $12.36 \pm 0.03$ after 180 days, $11.06 \pm 0.02$, $12.56 \pm 0.03,13.74 \pm 0.02$ and $12.53 \pm 0.02$, after 210 days and $11.01 \pm 0.01,12.93 \pm 0.05$, $13.98 \pm 0.05$ and $12.75 \pm 0.02$ after 240 days of study, respectively,. There was a significant change $(\mathrm{P}<0.01)$ in Calcium in treated groups as compared to control group (Table 01 and Figure 01).

The mean values of Phosphorus of T0, T1, T2 and T3 were $11.79 \pm 0.01, \quad 12.24 \pm 0.02$, $12.82 \pm 0.01$ and $11.94 \pm 0.02$, after 30 days of study, $11.82 \pm 0.07,12.76 \pm 0.07,12.91 \pm 0.05$ and $12.11 \pm 0.04$, after 60 days $11.87 \pm 0.01$, $12.87 \pm 0.02,12.96 \pm 0.02$ and $12.33 \pm 0.01$, after 90 days $11.94 \pm 0.02,12.92 \pm 0.02,12.99 \pm 0.02$, and $12.68 \pm 0.01$, after 120 days $12.00 \pm 0.02$, $12.94 \pm 0.02,13.16 \pm 0.04$ and $12.82 \pm 0.05$, after 150 days $12.06 \pm 0.05,13.26 \pm 0.01,13.43 \pm 0.02$ and $13.03 \pm 0.01$ after 180 days $12.18 \pm 0.05$, $13.49 \pm 0.01,13.74 \pm 0.01$ and $13.35 \pm 0.05$, after 210 days $12.29 \pm 0.01,13.94 \pm 0.02,14.12 \pm 0.04$ and $13.80 \pm 0.01$, respectively, after 240 days of study. There was a significant change $(\mathrm{P}<0.01)$ in Phosphorus except for days 30, $60,90,150$ and 240 days where the level was significance was $\mathrm{P}<0.05$ in all treated groups as compared to control group (Table 01 and Figure 02).

There was a significant $(\mathrm{p}<0.01)$ increase in the serum calcium level in all the treated groups as compared to control groups for germinated maize. The phosphorus concentration was significant $(\mathrm{P}<0.05)$ in days $30,60,90,150$ and 240 and highly significant after 120, 180 and 210 days of study.

\section{Alkaline and Acid Phosphatase (ALP and $\mathrm{ACP}$ )}

The mean values of Alkaline phosphatase for T0, T1, T2 and T3 were 153.15 \pm 0.02 , $151.20 \pm 0.04,150.65 \pm 0.11$ and $151.80 \pm 0.04$, after 30 days of study, $153.30 \pm 0.07$, $150.77 \pm 0.04,150.35 \pm 0.03$ and $151.69 \pm 0.08$, after 60 days, $153.64 \pm 0.02, \quad 150 \pm 0.05$, $149.96 \pm 0.03$ and $151.37 \pm 0.04$, after 90 days, $153.79 \pm 0.04,150.29 \pm 0.04,149.54 \pm 0.07$, and $151.15 \pm 0.06$, after 120 days, $154.50 \pm 0.07$, $150.01 \pm 0.04,149.38 \pm 0.07$ and $150.78 \pm 0.05$, after 150 days, $153.77 \pm 0.04,149.83 \pm 0.04$, $149.04 \pm 0.02$ and $150.43 \pm 0.13$ after 180 days, $153.88 \pm 0.03,149.43 \pm 0.08,148.74 \pm 0.05$ and $150.02 \pm 0.10$, after 210 days and $153.80 \pm 0.07$, $149.28 \pm 0.08,148.23 \pm 0.05$ and $149.88 \pm 0.01$, after 240 days of study, respectively. There was a significant change $(\mathrm{P}<0.01)$ in ALP except for days 30,60 and 90 days where the level was significance was $\mathrm{P}<0.05$ in treated groups as compared to control group (Table 02 and Figure 03).

The mean values of Acid phosphatase were $7.87 \pm 0.03, \quad 7.57 \pm 0.12, \quad 6.69 \pm 0.01 \quad$ and $7.69 \pm 0.13$, after 30 days of study, $8.16 \pm 0.04$, $7.29 \pm 0.02,6.38 \pm 0.03$ and $7.16 \pm 0.07$, after 60 days $8.14 \pm 0.03,6.97 \pm 0.02,6.09 \pm 0.02$ and $7.05 \pm 0.03$, after 90 days $8.06 \pm 0.01$, $6.67 \pm 0.03,5.83 \pm 0.04$, and $6.81 \pm 0.04$, after 120 days $8.39 \pm 0.02,6.30 \pm 0.04,5.45 \pm 0.03$ and $6.64 \pm 0.02, \quad$ after $\quad 150 \quad$ days $8.40 \pm 0.02,6.04 \pm 0.04, \quad 5.31 \pm 0.05 \quad$ and $6.30 \pm 0.02$ after 180 days $8.42 \pm 0.01$, $5.67 \pm 0.02,5.16 \pm 0.02$ and $6.19 \pm 0.02$, after 210 days $\quad 8.19 \pm 0.02, \quad 5.46 \pm 0.02,5.06 \pm 0.03$ and $6.06 \pm 0.02$ after 240 days, respectively, for T0, $\mathrm{T} 1, \mathrm{~T} 2$ and $\mathrm{T} 3$. 
There was a significant change $(\mathrm{P}<0.01)$ in Acid phosphatase in treated groups as compared to control group (Table 02 and Figure 04). The present study revealed that there was a significant $(\mathrm{p}<0.05)$ decrease in plasma alkaline phosphatase after 30, 60 and 90 days and acid phosphatase after 30 days, and in remaining days the activities of these enzymes were highly significant $(\mathrm{P}<0.01)$ in all the treated groups.

\section{Total proteins, Total cholesterol and glucose}

The mean values of Total protein for T0, T1, T2 and T3 were 7.01 $\pm 0.03,8.42 \pm 0.01$, $9.13 \pm 0.03$ and $8.12 \pm 0.01$, after 30 days of study, $7.05 \pm 0.02,8.65 \pm 0.16,9.35 \pm 0.01$ and $8.40 \pm 0.05$, after 60 days, $7.90 \pm 0.02$, $8.87 \pm 0.03,9.30 \pm 0.04$ and $8.42 \pm 0.01$, after 90 days, $7.93 \pm 0.01,8.57 \pm 0.02,9.42 \pm 0.04$, and $8.33 \pm 0.01$, after 120 days, $8.09 \pm 0.02$, $8.38 \pm 0.01,9.42 \pm 0.04$ and $8.63 \pm 0.01$, after 150 days, $8.16 \pm 0.02,8.41 \pm 0.05,9.46 \pm 0.01$ and $8.69 \pm 0.02$ after 180 days, $8.21 \pm 0.01$, $8.70 \pm 0.04,9.52 \pm 0.05$ and $8.47 \pm 0.14$, after 210 days and $8.32 \pm 0.05,8.73 \pm 0.05,9.63 \pm 0.02$ and $8.51 \pm 0.04$ after 240 days of study, respectively. There was a highly significant change $(\mathrm{P}<0.01)$ in Total protein in treated groups as compared to control group (Table 03 and Figure 05).

The mean values of Total cholesterol for T0, $\mathrm{T} 1, \mathrm{~T} 2$ and $\mathrm{T} 3$ were 125.54 \pm 0.63 , $113.58 \pm 0.81,110.04 \pm 0.58$ and $113.72 \pm 0.02$, after 30 days of study, $129.86 \pm 0.23$, $113.98 \pm 0.06,112.16 \pm 0.21$ and $112.25 \pm 0.05$, after 60 days, $131.59 \pm 0.25,114.89 \pm 0.11$, $112.63 \pm 0.21$ and $112.12 \pm 0.05$, after 90 days, $112.64 \pm 0.27,114.99 \pm 0.07,113.73 \pm 0.23$, and $112.29 \pm 0.04$, after 120 days, $132.74 \pm 0.29$, $115.21 \pm 0.08,113.87 \pm 0.23$ and $112.89 \pm 0.07$, after 150 days, $133.79 \pm 0.29,115.91 \pm 0.08$, $114.33 \pm 0.23$ and $113.12 \pm 0.05$ after 180 days, $136.94 \pm 0.19,113.11 \pm 0.03,114.47 \pm 0.13$ and
$114.01 \pm 0.07$, after 210 days and $134.19 \pm 0.29$, $116.71 \pm 0.08,114.93 \pm 0.23$ and $114.12 \pm 0.07$ after 240 days of study, respectively. There was a significant change $(\mathrm{P}<0.01)$ in Total cholesterol in treated groups as compared to control group (Table 03 and Figure 06).

The mean values of Glucose were $185.68 \pm 0.04,189.31 \pm 0.05,191.76 \pm 0.02$ and $187.21 \pm 0.01$, after 30 days of study, $187.95 \pm 0.01,189.93 \pm 0.02,192.04 \pm 0.01$ and $189.12 \pm 0.03$, after 60 days, $186.31 \pm 0.04$, $191.43 \pm 0.02,193.96 \pm 0.01$ and $189.56 \pm 0.01$, after 90 days, $187.03 \pm 0.03,187.03 \pm 0.03$, $195.10 \pm 0.02$, and $189.52 \pm 0.01$, after 120 days, $187.03 \pm 0.03,195.16 \pm 0.01,195.32 \pm 0.01$ and $189.52 \pm 0.01$, after 150 days, 189.72 \pm 0.05 , $195.46 \pm 0.04,196.01 \pm 0.14$ and $190.92 \pm 0.05$ after 180 days, $184.24 \pm 0.01,195.12 \pm 0.02$, $196.44 \pm 0.01$ and $191.24 \pm 0.02$, after 210 days and $190.74 \pm 0.01,195.64 \pm 0.01,196.72 \pm 0.02$ and 191.25 \pm 0.02 after 240 days of study, respectively, for $\mathrm{T} 0, \mathrm{~T} 1, \mathrm{~T} 2$ and $\mathrm{T} 3$ groups.

There was a significant change $(\mathrm{P}<0.01)$ in Glucose in treated groups as compared to control group (Table 03 and Figure 07).

The present study revealed a highly significant $(p<0.01)$ increase in the level of serum total protein in the entire period of the present study but the levels of total cholesterol was significant $(\mathrm{p}<0.05)$ decrease in all days of treatment except 210 and 240 days, where the decrease was highly significant $(\mathrm{p}<0.01)$.

\section{Calcium and Phosphorus}

There was a significant $(\mathrm{p}<0.01)$ increase in the serum calcium level in all the treated groups as compared to control groups for germinated maize. The phosphorus concentration was significant $(\mathrm{P}<0.05)$ in days 30, 60, 90, 150 and 240 and highly significant after 120, 180 and 210 days of study. This indicated that germinated maize in feed may 
have increased activity of phytase enzyme, which may be capable of releasing of calcium and inorganic phosphorus by its action phytic phosphorus (Bound form of phosphorus). So this inorganic phosphorus readily available for utilization by the body along with increased availability of bivalent ions and trivalent ions like calcium, magnesium, iron and zinc etc. for the growth and other metabolic activities of the body.

Table.1 Effect of germinated maize on biochemical parameters in Kadaknath after 30 to 240 days of study (Mean \pm SE)

\begin{tabular}{|c|c|c|c|c|c|c|c|c|}
\hline Calcium(mg/dl) & $\begin{array}{l}30 \\
\text { Days }\end{array}$ & $\begin{array}{l}60 \\
\text { Days }\end{array}$ & $\begin{array}{l}90 \\
\text { Days }\end{array}$ & $\begin{array}{l}120 \\
\text { Days }\end{array}$ & $\begin{array}{l}150 \\
\text { Days }\end{array}$ & $\begin{array}{l}180 \\
\text { Days }\end{array}$ & $\begin{array}{l}210 \\
\text { Days }\end{array}$ & $\begin{array}{l}240 \\
\text { Days }\end{array}$ \\
\hline T0 & $\begin{array}{l}10.06^{\mathrm{a}} \\
\pm 0.01\end{array}$ & $\begin{array}{l}10.72^{\mathrm{a}} \\
\pm 0.05\end{array}$ & $\begin{array}{l}10.97^{\mathrm{a}} \\
\pm 0.02\end{array}$ & $\begin{array}{l}10.33^{\mathrm{a}} \\
\pm 0.01\end{array}$ & $\begin{array}{l}10.32^{\mathrm{a}} \\
\pm 0.01\end{array}$ & $\begin{array}{l}10.63^{\mathrm{a}} \\
\pm 0.01\end{array}$ & $\begin{array}{l}11.06^{\mathrm{a}} \\
\pm 0.02\end{array}$ & $\begin{array}{l}11.01^{\mathrm{a}} \\
\pm 0.01\end{array}$ \\
\hline T1 & $\begin{array}{l}11.36^{c} \\
\pm 0.01\end{array}$ & $\begin{array}{l}11.63^{\mathrm{c}} \\
\pm 0.07\end{array}$ & $\begin{array}{l}11.93^{\mathrm{b}} \\
\pm 0.01\end{array}$ & $\begin{array}{l}12.03^{c} \\
\pm 0.04\end{array}$ & $\begin{array}{l}12.15^{c} \\
\pm 0.10\end{array}$ & $\begin{array}{l}12.32^{c} \\
\pm 0.05\end{array}$ & $\begin{array}{l}12.56 \\
\pm 0.04\end{array}$ & $\begin{array}{l}12.93^{\mathrm{c}} \\
\pm 0.05\end{array}$ \\
\hline $\mathbf{T} 2$ & $\begin{array}{l}12.36^{\mathrm{d}} \\
\pm 0.02\end{array}$ & $\begin{array}{l}12.44^{\mathrm{d}} \\
\pm 0.05\end{array}$ & $\begin{array}{l}12.74^{\mathrm{d}} \\
\pm 0.01\end{array}$ & $\begin{array}{l}12.96^{\mathrm{c}} \\
\pm 0.01\end{array}$ & $\begin{array}{l}13.09^{c} \\
\pm 0.02\end{array}$ & $\begin{array}{l}13.34^{\mathrm{d}} \\
\pm 0.01\end{array}$ & $\begin{array}{l}13.74^{\mathrm{d}} \\
\pm 0.02\end{array}$ & $\begin{array}{l}13.98^{d} \\
\pm 0.04\end{array}$ \\
\hline T3 & $\begin{array}{l}11.03^{b} \\
\pm 0.05^{6}\end{array}$ & $\begin{array}{l}11.31^{b} \\
\pm 0.03\end{array}$ & $\begin{array}{l}11.66^{c} \\
\pm 0.01\end{array}$ & $\begin{array}{l}11.88^{b} \\
\pm 0.02\end{array}$ & $\begin{array}{l}12.12^{b} \\
\pm 0.01\end{array}$ & $\begin{array}{l}12.36^{b} \\
\pm 0.03\end{array}$ & $\begin{array}{l}12.53^{b} \\
\pm 0.02\end{array}$ & $\begin{array}{l}12.75 \\
\pm 0.02\end{array}$ \\
\hline Phosphorus(mg/dl) & $* *$ & $* *$ & $* *$ & $* *$ & $* *$ & $* *$ & $* *$ & $* *$ \\
\hline T0 & $\begin{array}{l}11.79^{\mathrm{a}} \\
\pm 0.01\end{array}$ & $\begin{array}{l}11.82^{\mathrm{a}} \\
\pm 0.007\end{array}$ & $\begin{array}{l}11.87^{\mathrm{a}} \\
\pm 0.01\end{array}$ & $\begin{array}{l}11.94^{\mathrm{a}} \\
\pm 0.02\end{array}$ & $\begin{array}{l}12.00^{\mathrm{a}} \\
\pm 0.02\end{array}$ & $\begin{array}{l}12.06^{\mathrm{a}} \\
\pm 0.05\end{array}$ & $\begin{array}{l}12.18^{\mathrm{a}} \\
\pm 0.05\end{array}$ & $\begin{array}{l}12.29^{\mathrm{a}} \\
\pm 0.01\end{array}$ \\
\hline T1 & $\begin{array}{l}12.24^{c} \\
\pm 0.02\end{array}$ & $\begin{array}{l}12.76^{\mathrm{c}} \\
\pm 0.02\end{array}$ & $\begin{array}{l}12.87^{\mathrm{c}} \\
\pm 0.02\end{array}$ & $\begin{array}{l}12.92^{c} \\
\pm 0.02^{c}\end{array}$ & $\begin{array}{l}12.94^{b c} \\
\pm 0.02\end{array}$ & $\begin{array}{l}13.26^{\mathrm{c}} \\
\pm 0.01\end{array}$ & $\begin{array}{l}13.49^{c} \\
\pm 0.01\end{array}$ & $\begin{array}{l}13.94^{\mathrm{c}} \\
\pm 0.02^{2}\end{array}$ \\
\hline $\mathbf{T} 2$ & $\begin{array}{l}12.82^{c} \\
\pm 0.01\end{array}$ & $\begin{array}{l}12.91^{\mathrm{d}} \\
\pm 0.05\end{array}$ & $\begin{array}{l}12.96^{\mathrm{c}} \\
\pm 0.02\end{array}$ & $\begin{array}{l}12.99^{d} \\
\pm 0.02\end{array}$ & $\begin{array}{l}13.16^{c} \\
\pm 0.04\end{array}$ & $\begin{array}{l}13.43^{d} \\
\pm 0.02\end{array}$ & $\begin{array}{l}13.74^{\mathrm{d}} \\
\pm 0.01\end{array}$ & $\begin{array}{l}14.12^{d} \\
\pm 0.04\end{array}$ \\
\hline T3 & $\begin{array}{l}11.94^{b} \\
\pm 0.02^{b}\end{array}$ & $\begin{array}{l}12.11^{b} \\
\pm 0.04\end{array}$ & $\begin{array}{l}12.33^{b} \\
\pm 0.01\end{array}$ & $\begin{array}{l}12.68^{\mathrm{b}} \\
\pm 0.01\end{array}$ & $\begin{array}{l}12.82^{b} \\
\pm 0.05\end{array}$ & $\begin{array}{l}13.03^{b} \\
\pm 0.01\end{array}$ & $\begin{array}{l}13.35^{\mathrm{b}} \\
\pm 0.05\end{array}$ & $\begin{array}{l}13.80^{\mathrm{b}} \\
\pm 0.01\end{array}$ \\
\hline & $*$ & $*$ & $*$ & $* *$ & $*$ & $* *$ & $* *$ & $*$ \\
\hline
\end{tabular}

**Shows Significance at $1 \%$ level as compared to control group $(\mathrm{P}<0.01)$

*Shows Significance at 5\% level as compared to control group $(\mathrm{P}<0.05)$ 
Table.2 Effect of germinated maize on biochemical parameters in Kadaknath after 30 to 240 days of study (Mean \pm SE)

\begin{tabular}{|c|c|c|c|c|c|c|c|c|}
\hline $\begin{array}{l}\text { Alkaline } \\
\text { phosphatase } \\
\text { (IU/l) }\end{array}$ & $\begin{array}{l}30 \\
\text { Days }\end{array}$ & $\begin{array}{l}60 \\
\text { Days }\end{array}$ & $\begin{array}{l}90 \\
\text { Days }\end{array}$ & $\begin{array}{l}120 \\
\text { Days }\end{array}$ & $\begin{array}{l}150 \\
\text { Days }\end{array}$ & $\begin{array}{l}180 \\
\text { Days }\end{array}$ & $\begin{array}{l}210 \\
\text { Days }\end{array}$ & $\begin{array}{l}240 \\
\text { Days }\end{array}$ \\
\hline T0 & $\begin{array}{l}153.15^{\mathrm{a}} \\
\pm 0.02\end{array}$ & $\begin{array}{l}153.30^{\mathrm{a}} \\
\pm 0.07\end{array}$ & $\begin{array}{l}153.64^{\mathrm{a}} \\
\pm 0.02\end{array}$ & $\begin{array}{l}153.79^{\mathrm{a}} \\
\pm 0.04\end{array}$ & $\begin{array}{l}154.50^{\mathrm{a}} \\
\pm 0.07\end{array}$ & $\begin{array}{l}153.77^{\mathrm{a}} \\
\pm 0.04\end{array}$ & $\begin{array}{l}153.88^{a} \\
\pm 0.03\end{array}$ & $\begin{array}{l}153.80^{a} \\
\pm 0.07\end{array}$ \\
\hline T1 & $\begin{array}{l}151.20^{b} \\
\pm 0.04\end{array}$ & $\begin{array}{l}150.77^{c} \\
\pm 0.04\end{array}$ & $\begin{array}{l}150.56^{b} \\
\pm 0.05\end{array}$ & $\begin{array}{l}150.29^{c} \\
\pm 0.04\end{array}$ & $\begin{array}{l}150.01^{\mathrm{a}} \\
\pm 0.04\end{array}$ & $\begin{array}{l}149.83^{b} \\
\pm 0.04\end{array}$ & $\begin{array}{l}149.43^{b} \\
\pm 0.01\end{array}$ & $\begin{array}{l}149.28^{b} \\
\pm 0.08\end{array}$ \\
\hline $\mathbf{T} 2$ & $\begin{array}{l}150.65^{c} \\
\pm 0.11\end{array}$ & $\begin{array}{l}150.35^{c} \\
\pm 0.03\end{array}$ & $\begin{array}{l}149.96^{c} \\
\pm 0.03\end{array}$ & $\begin{array}{l}149.54^{\mathrm{c}} \\
\pm 0.07\end{array}$ & $\begin{array}{l}149.38^{b} \\
\pm 0.07\end{array}$ & $\begin{array}{l}149.04^{c} \\
\pm 0.02\end{array}$ & $\begin{array}{l}148.74^{\mathrm{c}} \\
\pm 0.05\end{array}$ & $\begin{array}{l}148.23^{c} \\
\pm 0.05\end{array}$ \\
\hline T3 & $\begin{array}{l}151.80^{\mathrm{a}} \\
\pm 0.04\end{array}$ & $\begin{array}{l}151.69^{b} \\
\pm 0.08\end{array}$ & $\begin{array}{l}151.37^{b} \\
\pm 0.04\end{array}$ & $\begin{array}{l}151.15^{b} \\
\pm 0.06\end{array}$ & $\begin{array}{l}150.78^{a} \\
\pm 0.05\end{array}$ & $\begin{array}{l}150.43^{b} \\
\pm 0.13\end{array}$ & $\begin{array}{l}150.02^{b} \\
\pm 0.10\end{array}$ & $\begin{array}{l}149.88^{b} \\
\pm 0.01\end{array}$ \\
\hline $\begin{array}{l}\text { Acid } \\
\text { phosphatase } \\
\text { (IU/l) }\end{array}$ & $*$ & $*$ & $*$ & $* *$ & $* *$ & $* *$ & $* *$ & $* *$ \\
\hline T0 & $\begin{array}{l}7.87^{\mathrm{a}} \\
\pm 0.03\end{array}$ & $\begin{array}{l}8.16^{\mathrm{a}} \\
\pm 0.04\end{array}$ & $\begin{array}{l}8.14^{\mathrm{a}} \\
\pm 0.03\end{array}$ & $\begin{array}{l}8.06^{a} \\
\pm 0.01\end{array}$ & $\begin{array}{l}8.39^{\mathrm{a}} \\
\pm 0.02\end{array}$ & $\begin{array}{l}8.40^{\mathrm{a}} \\
\pm 0.02\end{array}$ & $\begin{array}{l}8.42^{\mathrm{a}} \\
\pm 0.01\end{array}$ & $\begin{array}{l}8.19^{\mathrm{a}} \\
\pm 0.02\end{array}$ \\
\hline T1 & $\begin{array}{l}7.57^{\mathrm{a}} \\
\pm 0.12\end{array}$ & $\begin{array}{l}7.29^{b} \\
\pm 0.02\end{array}$ & $\begin{array}{l}6.97^{b} \\
\pm 0.02\end{array}$ & $\begin{array}{l}6.67^{b} \\
\pm 0.03\end{array}$ & $\begin{array}{l}6.30^{b} \\
\pm 0.04\end{array}$ & $\begin{array}{l}6.04^{b} \\
\pm 0.04\end{array}$ & $\begin{array}{l}5.67^{b} \\
\pm 0.02\end{array}$ & $\begin{array}{l}5.46^{c} \\
\pm 0.02\end{array}$ \\
\hline $\mathbf{T} 2$ & $\begin{array}{l}6.69^{b} \\
\pm 0.01\end{array}$ & $\begin{array}{l}6.38^{d} \\
\pm 0.03\end{array}$ & $\begin{array}{l}6.09^{d} \\
\pm 0.02\end{array}$ & $\begin{array}{l}5.83^{d} \\
\pm 0.04\end{array}$ & $\begin{array}{l}5.45^{c} \\
\pm 0.03\end{array}$ & $\begin{array}{l}5.31^{d} \\
\pm 0.05\end{array}$ & $\begin{array}{l}5.16^{\mathrm{c}} \\
\pm 0.02\end{array}$ & $\begin{array}{l}5.06^{d} \\
\pm 0.03\end{array}$ \\
\hline T3 & $\begin{array}{l}7.69^{\mathrm{a}} \\
\pm 0.13\end{array}$ & $\begin{array}{l}7.16^{c} \\
\pm 0.07\end{array}$ & $\begin{array}{l}7.05^{c} \\
\pm 0.03\end{array}$ & $\begin{array}{l}6.81^{c} \\
\pm 0.04\end{array}$ & $\begin{array}{l}6.64^{c} \\
\pm 0.02\end{array}$ & $\begin{array}{l}6.30^{c} \\
\pm 0.02\end{array}$ & $\begin{array}{l}6.19^{c} \\
\pm 0.02\end{array}$ & $\begin{array}{l}6.06^{c} \\
\pm 0.02\end{array}$ \\
\hline & $*$ & $* *$ & $* *$ & $* *$ & $* *$ & $* *$ & $* *$ & $* *$ \\
\hline
\end{tabular}

**Shows Significance at $1 \%$ level as compared to control group $(\mathrm{P}<0.01)$

*Shows Significance at $5 \%$ level as compared to control group $(\mathrm{P}<0.05)$ 
Table.3 Effect of germinated maize on biochemical parameters in Kadaknath after 30 to 240 days of study (Mean \pm SE)

\begin{tabular}{|c|c|c|c|c|c|c|c|c|}
\hline $\begin{array}{l}\text { Total protein } \\
\text { (g/dl) }\end{array}$ & $\begin{array}{l}\text { 30 } \\
\text { Days }\end{array}$ & $\begin{array}{l}\text { 60 } \\
\text { Days }\end{array}$ & $\begin{array}{l}90 \\
\text { Days }\end{array}$ & $\begin{array}{c}120 \\
\text { Days }\end{array}$ & $\begin{array}{l}\text { 150 } \\
\text { Days }\end{array}$ & $\begin{array}{l}\text { 180 } \\
\text { Days }\end{array}$ & $\begin{array}{l}210 \\
\text { Days }\end{array}$ & $\begin{array}{l}240 \\
\text { Days }\end{array}$ \\
\hline T0 & $\begin{array}{l}7.01^{\mathrm{a}} \\
\pm 0.03\end{array}$ & $\begin{array}{l}7.05^{\mathrm{a}} \\
\pm 0.02\end{array}$ & $\begin{array}{l}7.90^{\mathrm{a}} \\
\pm 0.02\end{array}$ & $\begin{array}{l}7.93^{\mathrm{a}} \\
\pm 0.01\end{array}$ & $\begin{array}{l}8.09^{\mathrm{a}} \\
\pm 0.02\end{array}$ & $\begin{array}{l}8.16^{\mathrm{a}} \\
\pm 0.02\end{array}$ & $\begin{array}{l}8.21^{\mathrm{a}} \\
\pm 0.01\end{array}$ & $\begin{array}{l}8.32^{\mathrm{a}} \\
\pm 0.05\end{array}$ \\
\hline T1 & $\begin{array}{l}8.42 \mathrm{c} \\
\pm 0.01\end{array}$ & $\begin{array}{l}8.65^{b} \\
\pm 0.16\end{array}$ & $\begin{array}{l}8.87^{c} \\
\pm 0.03\end{array}$ & $\begin{array}{l}8.57^{\mathrm{c}} \\
\pm 0.02\end{array}$ & $\begin{array}{l}8.38^{b} \\
\pm 0.01\end{array}$ & $\begin{array}{l}8.41^{b} \\
\pm 0.05\end{array}$ & $\begin{array}{l}8.70^{\mathrm{c}} \\
\pm 0.04\end{array}$ & $\begin{array}{l}8.73^{c} \\
\pm 0.05\end{array}$ \\
\hline T2 & $\begin{array}{l}9.13^{\mathrm{d}} \\
\pm 0.03\end{array}$ & $\begin{array}{l}9.35^{\mathrm{c}} \\
\pm 0.01\end{array}$ & $\begin{array}{l}9.30^{\mathrm{d}} \\
\pm 0.004\end{array}$ & $\begin{array}{l}9.42^{\mathrm{d}} \\
\pm 0.04\end{array}$ & $\begin{array}{l}9.42^{\mathrm{d}} \\
\pm 0.04\end{array}$ & $\begin{array}{l}9.46^{\mathrm{d}} \\
\pm 0.01\end{array}$ & $\begin{array}{l}9.52^{\mathrm{d}} \\
\pm 0.05\end{array}$ & $\begin{array}{l}9.63^{b} \\
\pm 0.02\end{array}$ \\
\hline T3 & $\begin{array}{l}8.12^{b} \\
\pm 0.01\end{array}$ & $\begin{array}{l}8.40^{b} \\
\pm 0.05\end{array}$ & $\begin{array}{l}8.42^{b} \\
\pm 0.01\end{array}$ & $\begin{array}{l}8.33^{b} \\
\pm 0.01\end{array}$ & $\begin{array}{l}8.63^{c} \\
\pm 0.01\end{array}$ & $\begin{array}{l}8.69^{c} \\
\pm 0.02\end{array}$ & $\begin{array}{l}8.47^{b} \\
\pm 0.14\end{array}$ & $\begin{array}{l}8.51^{b} \\
\pm 0.04\end{array}$ \\
\hline $\begin{array}{l}\text { Total cholesterol } \\
\text { (mg/dl) }\end{array}$ & $* *$ & $* *$ & $* *$ & $* *$ & $* *$ & $* *$ & $* *$ & $* *$ \\
\hline T0 & $\begin{array}{l}125.54^{\mathrm{a}} \\
\pm 0.63\end{array}$ & $\begin{array}{l}129.86^{\mathrm{a}} \\
\pm 0.23\end{array}$ & $\begin{array}{l}131.59^{\mathrm{a}} \\
\pm 0.25\end{array}$ & $\begin{array}{l}122.64^{\mathrm{a}} \\
\pm 0.27\end{array}$ & $\begin{array}{l}132.74^{\mathrm{a}} \\
\pm 0.29\end{array}$ & $\begin{array}{l}133.79^{\mathrm{a}} \\
\pm 0.29\end{array}$ & $\begin{array}{l}136.94^{\mathrm{a}} \\
\pm 0.19\end{array}$ & $\begin{array}{l}134.19^{\mathrm{a}} \\
\pm 0.29\end{array}$ \\
\hline T1 & $\begin{array}{l}113.58^{\mathrm{a}} \\
\pm 0.81\end{array}$ & $\begin{array}{l}113.98^{b} \\
\pm 0.06\end{array}$ & $\begin{array}{l}114.89^{\mathrm{c}} \\
\pm 0.11\end{array}$ & $\begin{array}{l}114.99^{\mathrm{c}} \\
\pm 0.07\end{array}$ & $\begin{array}{l}115.21^{\mathrm{c}} \\
\pm 0.08\end{array}$ & $\begin{array}{l}115.91^{\mathrm{c}} \\
\pm 0.08\end{array}$ & $\begin{array}{l}113.11^{\mathrm{a}} \\
\pm 0.03\end{array}$ & $\begin{array}{l}116.71^{\mathrm{b}} \\
\pm 0.08\end{array}$ \\
\hline T2 & $\begin{array}{l}110.04^{b} \\
\pm 0.58\end{array}$ & $\begin{array}{l}112.16^{\mathrm{c}} \\
\pm 0.21\end{array}$ & $\begin{array}{l}112.63^{\mathrm{b}} \\
\pm 0.21\end{array}$ & $\begin{array}{ll} & 113.73^{b} \\
\pm & 0.23\end{array}$ & $\begin{array}{l}113.87^{\mathrm{b}} \\
\pm 0.23\end{array}$ & $\begin{array}{l}114.33^{b} \\
\pm 0.23\end{array}$ & $\begin{array}{l}114.47^{\mathrm{b}} \\
\pm 0.13\end{array}$ & $\begin{array}{l}114.93^{\mathrm{a}} \\
\pm 0.23\end{array}$ \\
\hline T3 & $\begin{array}{l}113.72 \\
\mathrm{~b} \\
\pm 0.02\end{array}$ & $\begin{array}{l}112.25 \\
{ }^{b} \pm 0.05\end{array}$ & $\begin{array}{l}112.12 \\
\mathrm{~b} \\
\pm 0.05\end{array}$ & $\begin{array}{l}112.29^{\mathrm{a}} \\
\pm 0.04\end{array}$ & $\begin{array}{l}112.89 \\
\mathrm{a} \pm 0.07\end{array}$ & $\begin{array}{l}113.12 \\
\mathrm{a} \pm 0.05\end{array}$ & $\begin{array}{l}114.01 \\
\mathrm{~b} \\
\pm 0.07\end{array}$ & $\begin{array}{l}114.12 \\
\mathrm{a} \\
\pm 0.07\end{array}$ \\
\hline & * & * & $*$ & * & $*$ & $*$ & $* *$ & $* *$ \\
\hline \multicolumn{9}{|l|}{ Glucose (mg/dl) } \\
\hline T0 & $\begin{array}{l}185.68^{\mathrm{a}} \\
\pm 0.04\end{array}$ & $\begin{array}{l}187.95^{\mathrm{a}} \\
\pm 0.01\end{array}$ & $\begin{array}{l}186.31^{\mathrm{a}} \\
\pm 0.04\end{array}$ & $\begin{array}{l}187.03^{a} \\
\pm 0.03\end{array}$ & $\begin{array}{l}187.03^{\mathrm{a}} \\
\pm 0.03\end{array}$ & $\begin{array}{l}189.72^{b} \\
\pm 0.05\end{array}$ & $\begin{array}{l}184.24^{\mathrm{a}} \\
\pm 0.01\end{array}$ & $\begin{array}{l}190.74^{\mathrm{a}} \\
\pm 0.01\end{array}$ \\
\hline T1 & $\begin{array}{l}189.31^{\mathrm{c}} \\
\pm 0.05\end{array}$ & $\begin{array}{l}189.93^{\mathrm{a}} \\
\pm 0.02\end{array}$ & $\begin{array}{l}191.43^{\mathrm{c}} \\
\pm 0.02\end{array}$ & $\begin{array}{l}187.03^{a} \\
\pm 0.03\end{array}$ & $\begin{array}{l}195.16^{\mathrm{c}} \\
\pm 0.01\end{array}$ & $\begin{array}{l}195.46^{\mathrm{c}} \\
\pm 0.04\end{array}$ & $\begin{array}{l}195.12^{c} \\
\pm 0.02\end{array}$ & $\begin{array}{l}195.64^{\mathrm{c}} \\
\pm 0.01\end{array}$ \\
\hline T2 & $\begin{array}{l}191.76^{\mathrm{d}} \\
\pm 0.02\end{array}$ & $\begin{array}{l}192.04^{\mathrm{b}} \\
\pm 0.01\end{array}$ & $\begin{array}{l}193.96^{\mathrm{d}} \\
\pm 0.01\end{array}$ & $\begin{array}{l}195.10^{\mathrm{d}} \\
\pm 0.02\end{array}$ & $\begin{array}{l}195.32^{\mathrm{d}} \\
\pm 0.01\end{array}$ & $\begin{array}{l}196.01^{\mathrm{d}} \\
\pm 0.14\end{array}$ & $\begin{array}{l}196.44^{\mathrm{d}} \\
\pm 0.01\end{array}$ & $\begin{array}{l}196.72^{\mathrm{d}} \\
\pm 0.02\end{array}$ \\
\hline T3 & $\begin{array}{l}187.21^{\mathrm{b}} \\
\pm 0.01\end{array}$ & $\begin{array}{l}189.12^{\mathrm{a}} \\
\pm 0.03\end{array}$ & $\begin{array}{l}189.56^{\mathrm{b}} \\
\pm 0.01\end{array}$ & $\begin{array}{l}189.52^{\mathrm{b}} \\
\pm 0.01\end{array}$ & $\begin{array}{l}189.52^{\mathrm{b}} \\
\pm 0.01\end{array}$ & $\begin{array}{l}190.92^{\mathrm{b}} \\
\pm 0.05\end{array}$ & $\begin{array}{l}191.24^{\mathrm{b}} \\
\pm 0.02\end{array}$ & $\begin{array}{l}191.25^{\mathrm{b}} \\
\pm 0.02\end{array}$ \\
\hline & $* *$ & $*$ & $* *$ & $* *$ & $* *$ & $* *$ & $* *$ & $*$ \\
\hline
\end{tabular}

**Shows Significance at $1 \%$ level as compared to control group $(\mathrm{P}<0.01)$

*Shows Significance at $5 \%$ level as compared to control group $(\mathrm{P}<0.05)$ 
Fig.1 Graph showing effect of germinated maize on calcium $(\mathrm{mg} / \mathrm{dl})$ in kadaknath

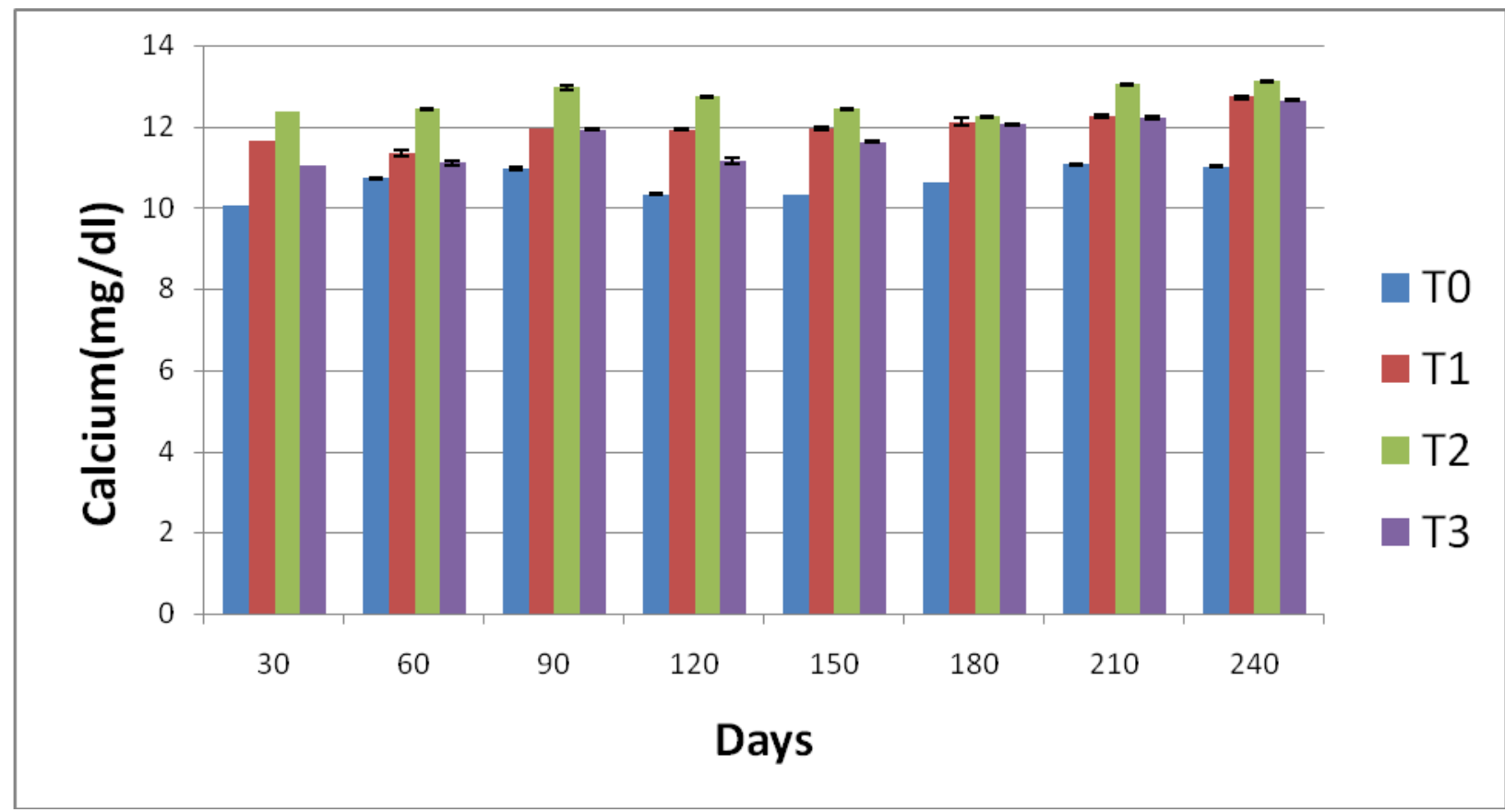

The value with the different superscripts in a row are different significantly between groups. The value with the no superscripts in a row are having no significant relationship

Fig.2 Graph showing effect of germinated maize on phosphrus (mg/dl) in kadaknath

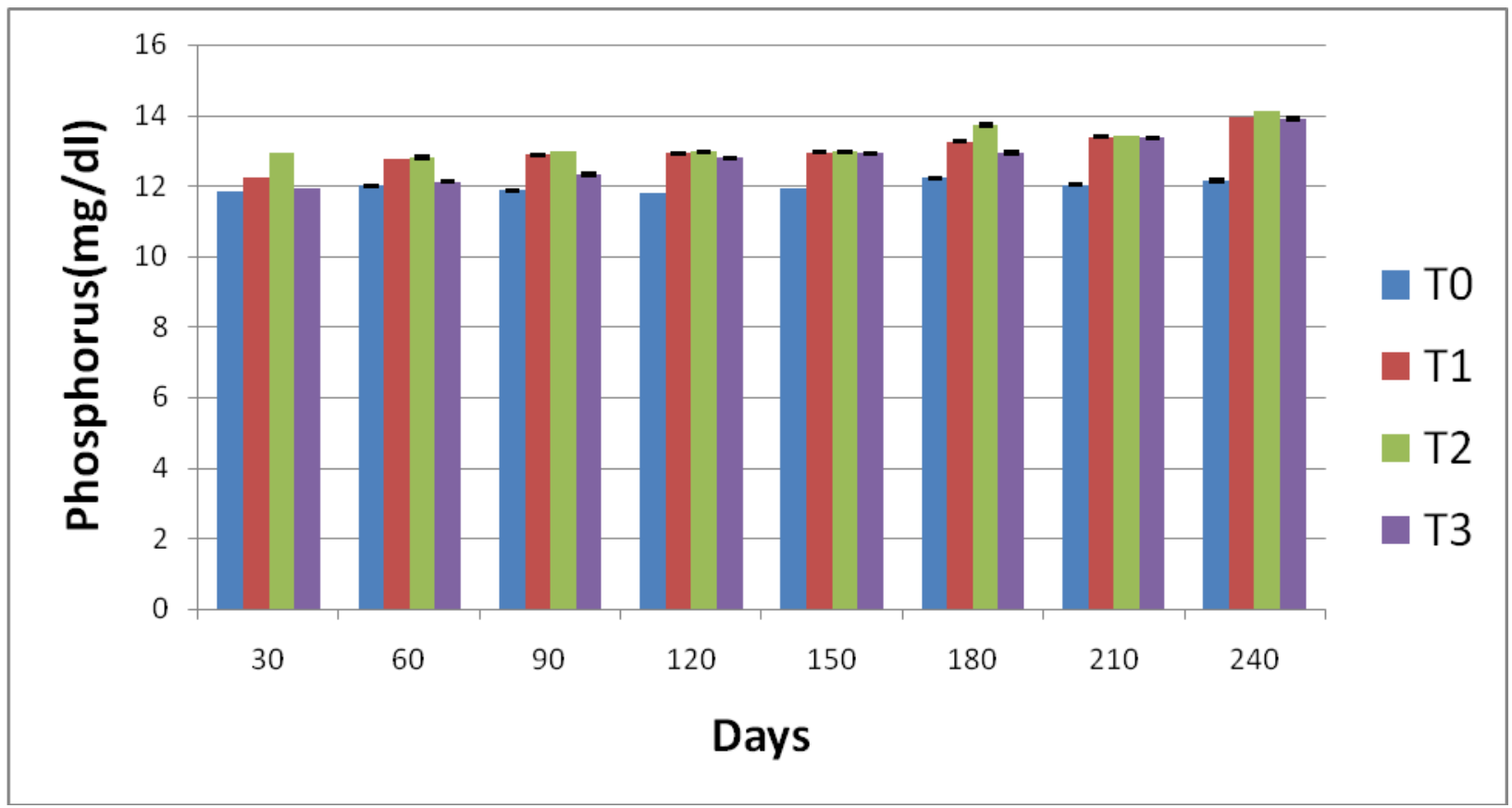

The value with the different superscripts in a row are different significantly between groups. The value with the no superscripts in a row are having no significant relationship. 
Fig.3 Graph showing effect of germinated maize on ALP (IU/L) in kadaknath

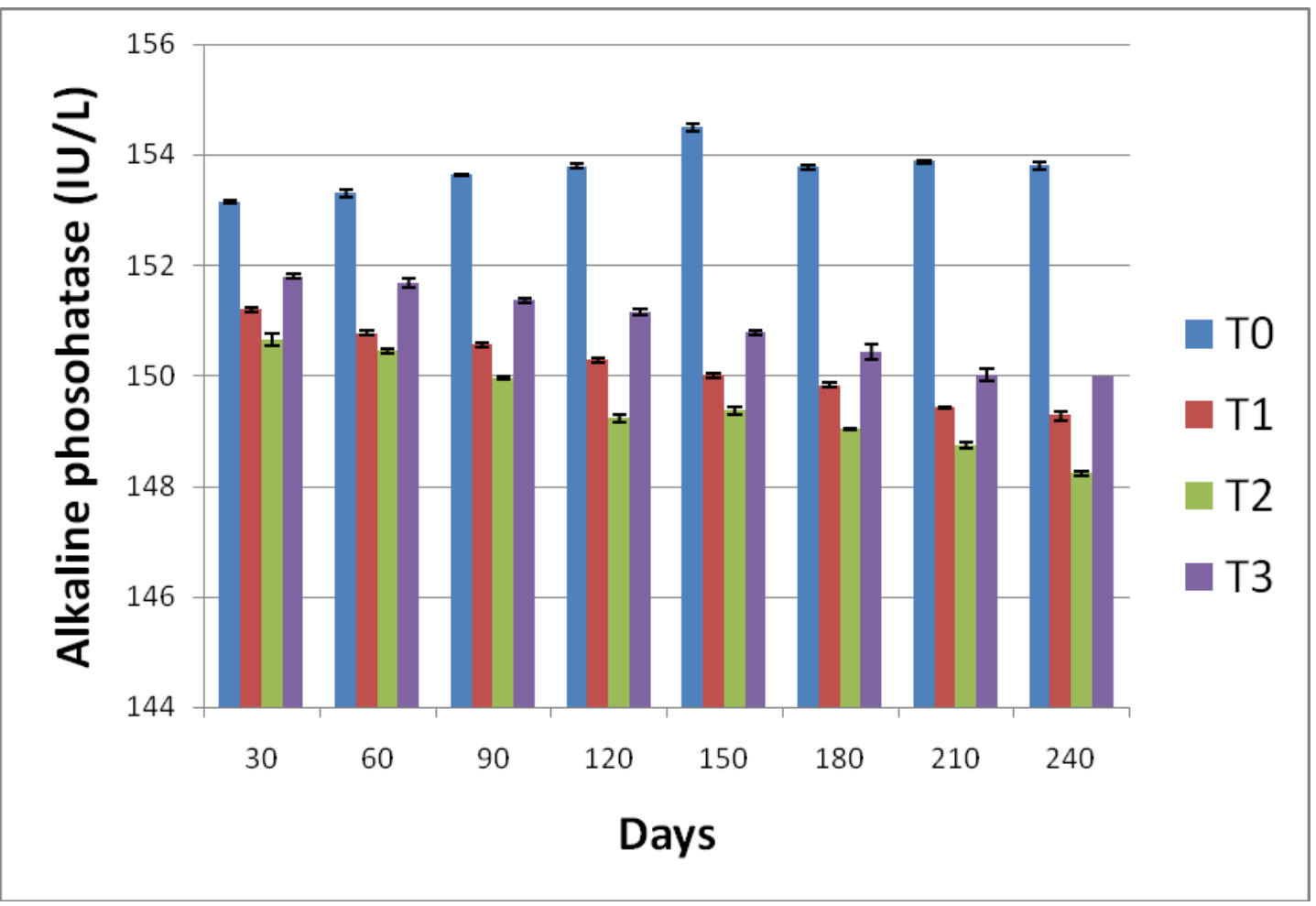

Fig.4 Graph showing effect of germinated maize on ACP (IU/L) in kadaknath

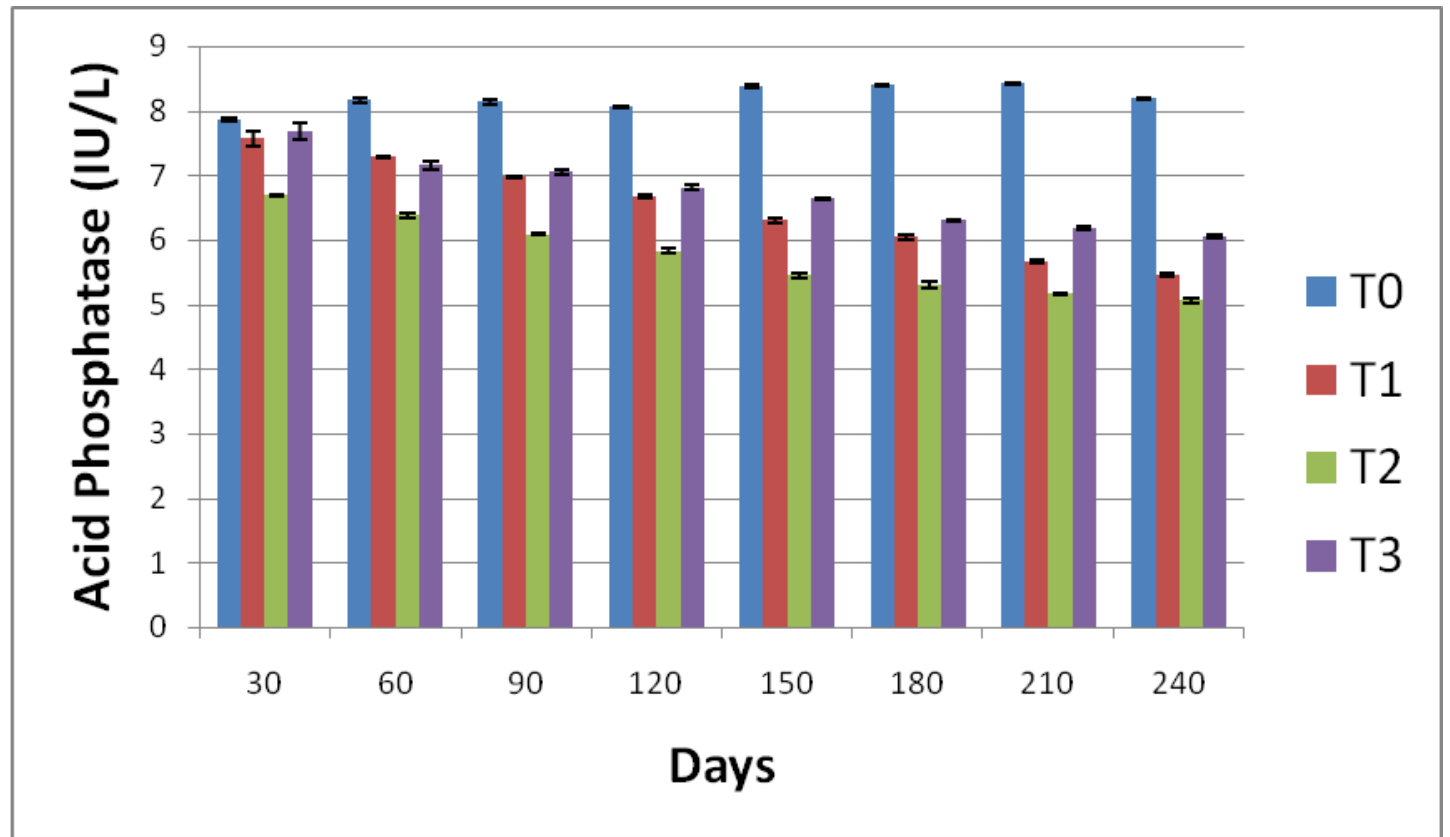

The value with the different superscripts in a row are different significantly between groups. The value with the no superscripts in a row are having no significant relationship 
Fig.5 Graph showing effect of germinated maize on Total protein $(\mathrm{g} / \mathrm{dl})$ in kadaknath

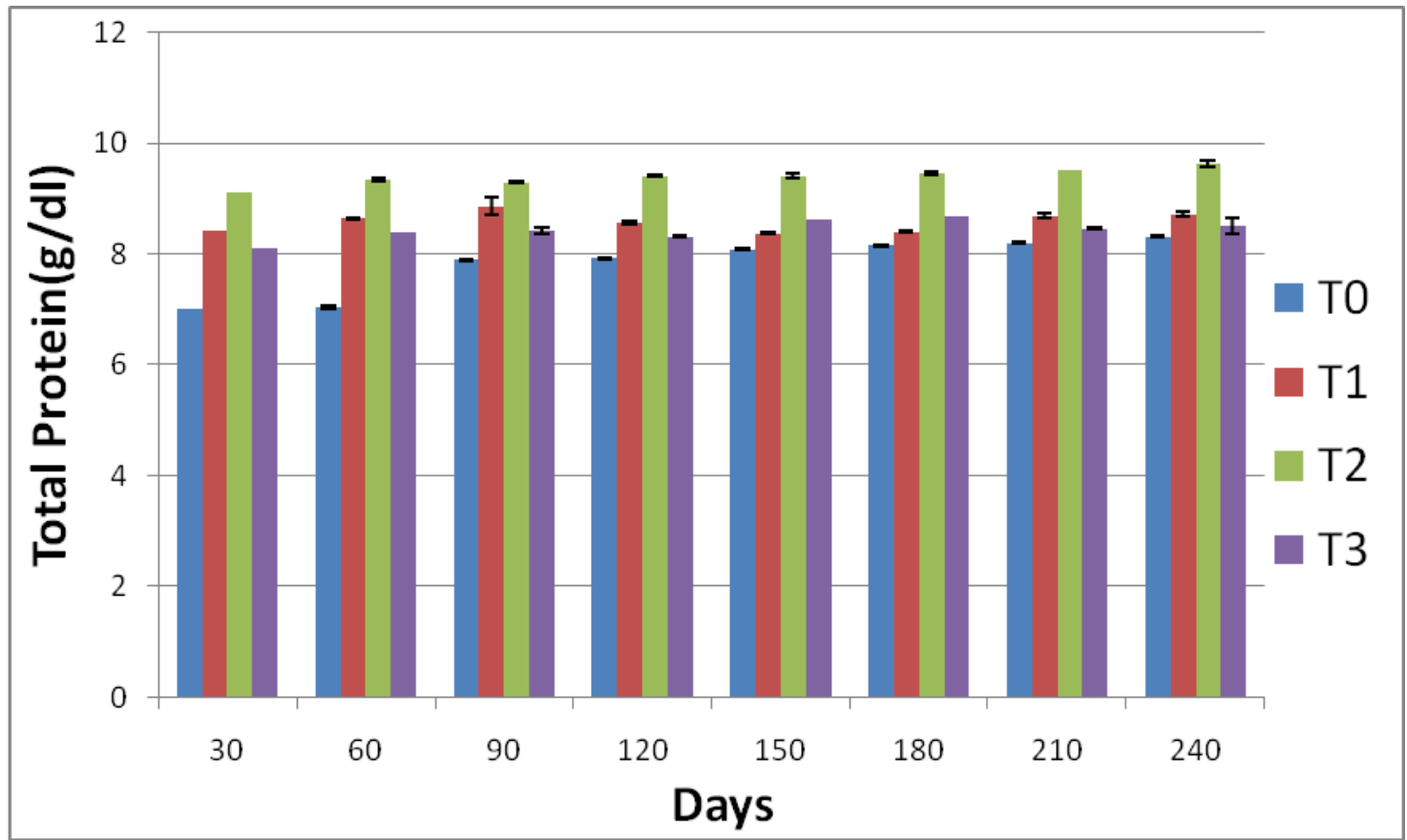

Fig.6 Graph showing effect of germinated maize on Total protein $(\mathrm{g} / \mathrm{dl})$ in kadaknath

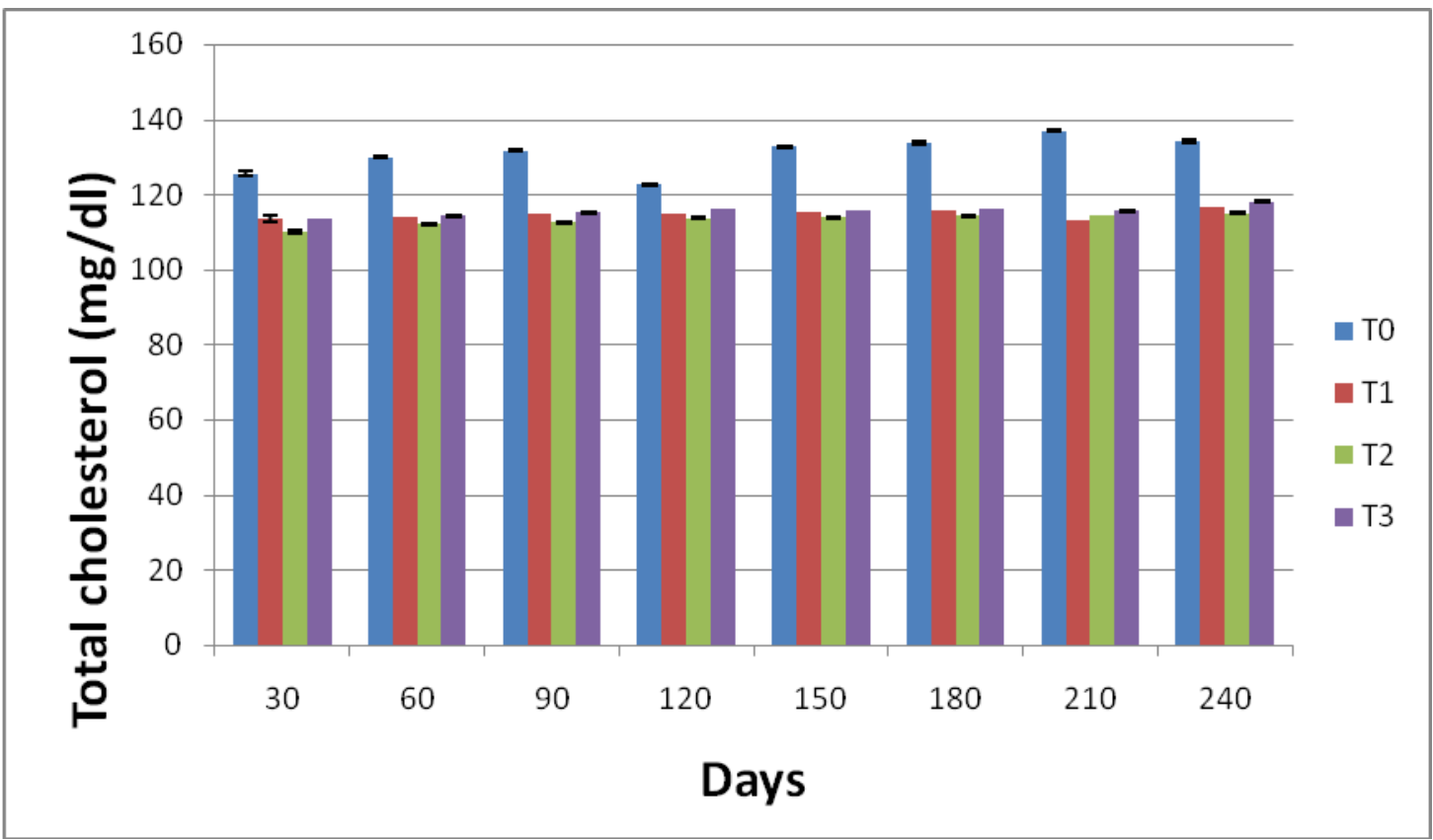


Fig.7 Graph showing effect of germinated maize on glucose (mg/dl) in kadaknath

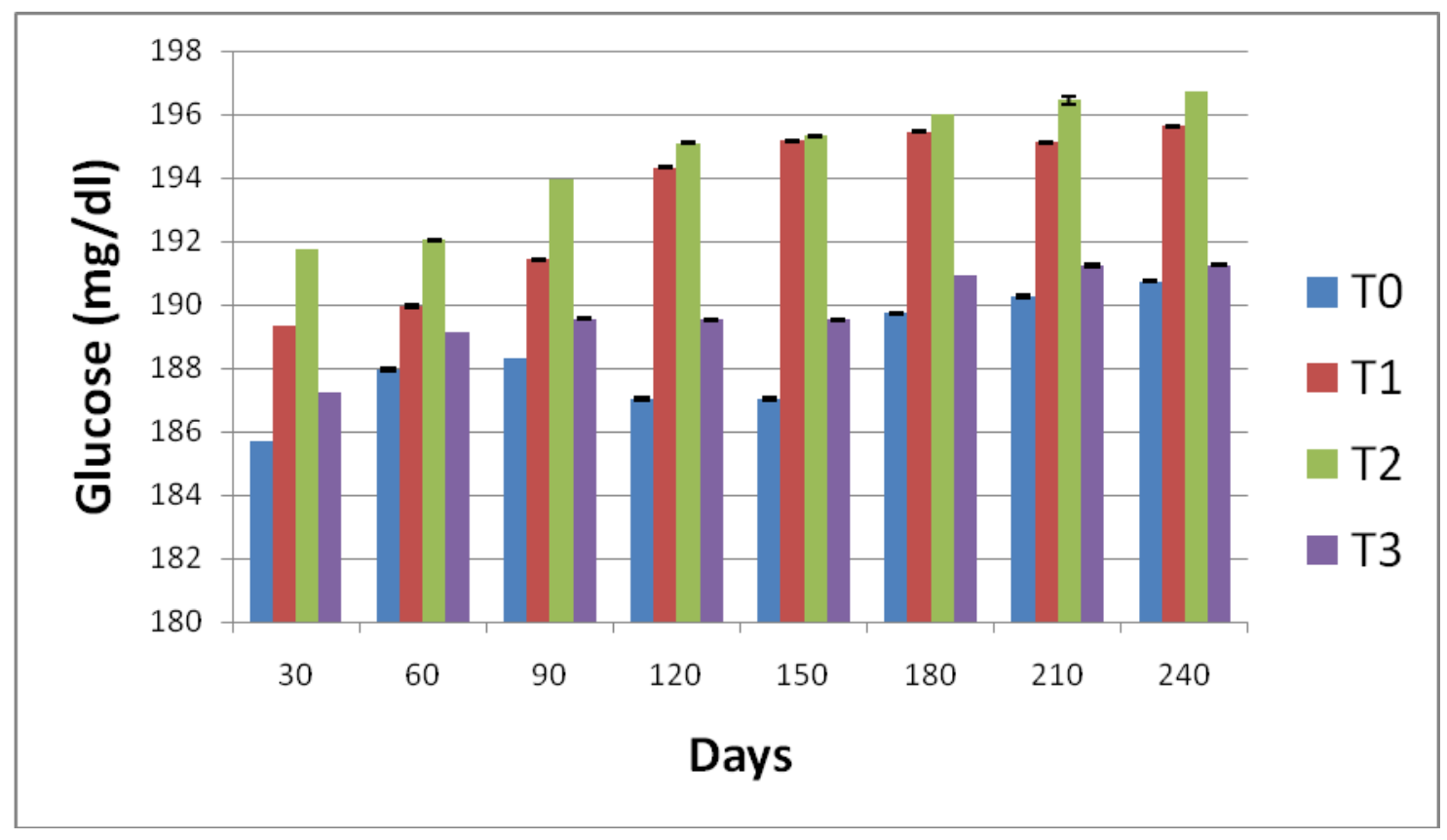

Similar observations were also reported by Singh and Khatta (2003), Jain (2008), Osman (2009), Prajapati (2010), Kwari et al., (2012), Jamre (2015), Gautam et al., (2017), and Khan et al., (2017).

\section{Acid and Alkaline Phosphatase}

The present study revealed that there was a significant $(\mathrm{p}<0.05)$ decrease in plasma alkaline phosphatase after 30,60 and 90 days and acid phosphatase after 30 days, and in remaining days the activities of these enzymes were highly significant $(\mathrm{P}<0.01)$ in all the treated groups. This decrease in acid and alkaline phosphatase enzymes may indicated that sprouted grains given to Kadaknath chicks may increase the activity of some enzymes like phytase, resulted in increased plasma concentration of phosphorus and calcium through liberation of phosphorus, bivalent and trivalent ions, which are readily available to the chicks. All the results reported in the present study were in accordance with the observations reported by Viveros et al., (2002), Fanimo and Akinola (2006), Jain
(2008), Prajapati (2010), Jamre (2015), Gautam et al., (2017) and Khan et al., (2017). In contrast non significant effect was noted by Onifade et al., (1999). Orban et al., (1999) observed that the phosphorus excretion was very much decreased and the phytate phosphorus utilization was increased significantly.

\section{Total proteins, Total cholesterol and glucose}

The present study revealed a highly significant $(p<0.01)$ increase in the level of serum total protein in the entire period of the present study but the levels of total cholesterol was significant $(p<0.05)$ decrease in all days of treatment except 210 and 240 days, where the decrease was highly significant $(p<0.01)$. Certain workers (Lee et al., 2010, Demonty et al., 2003) showed that the reduction of the blood's total and LDL cholesterol concentrations with the consumption of products that contain soy protein has been shown repeatedly in humans and several animal models The exact component that is 
responsible for this action has yet to be identified. Isoflavone have been proposed to be the active ingredient that is responsible for the hypocholesterolemic effects of soy. One of the study suggested that isoflavonerich soy protein is considerably more effective than isoflavone-depleted soy protein, though this finding is controversial. The cholesterollowering effect is one of several proposed mechanisms by which soy reduces the risk of heart disease (Gardner et al., 2001). During germination protease enzymes are activated that convert the protein polymers into amino acids and small peptides (Shewry et al., 1995). These enzymes convert the complex compounds of protein into albumin and globulin thus, improve the quality protein. They also improve the lysine content of grains (Chavan and Kadam, 1989). Activation of amylase and lipase during germination increases the sugar and essential fatty acid content of grains (Chavan and Kadam, 1989).

The glucose concentrations were highly significant $(\mathrm{p}<0.01)$ of the entire study period in germinated maize supplemented group except on Days 60 and 240 where it was significant at $p<0.05$. The observations for total proteins, total cholesterol and glucose in the present study were in accordance with the findings of El-Deek et al., (2009), Osman (2009), Lee et al., (2010), Gautam et al., (2017) and Khan et al., (2017). On the contrary, Jain (2008), Manwar and mandal (2009) and Prajapati (2010) found no significant effect on the above parameters in treated groups as compare to control.

From the present study, it is concluded that supplementation of germinated maize in feed of kadaknath layer birds had very good effect on availability of protein, calcium, and phosphorus, along with certain enzymes and also there was a decreasing trend in total cholesterol, specially at the level of $50 \%$ and $75 \%$ germinated maize as compared to the non-germinated maize based diets. Germination of grains may cause activation of different enzymes eg. protease, which convert the protein polymers into amino acid and small peptides. They may also improve the lysine content of grains and make feed more nutritive and palatable to Kadaknath. Phytase made available the bi and tri- valant ions like calcium and phosphorus and beta glucanase affects the availability of energy by fiber digestion.

\section{References}

Chavan, J. and Kadam, S.S. (1989). Nutritional improvement of cereals by sprouting. Critical Reviews in Food Science and Nutrition, 28(5): 401-437.

Demonty, I., Benoit L., and Peter JH Jones. (2003) Role of Isoflavones in the Hypocholesterolemic Effect of Soy. Nutrition Reviews 61(6):189-203.

El-Deek, A.A., Osman, M., Yakout, H.M. and Yahya, E. (2009). Response of broilers to microbial phytase supplementation as influenced by dietary corn gluten meal levels. Egyptian Poultry Science, 29(1):77-97

Fanimo, A.O. and Akinola, O.S. (2006). Response of broiler chicken to raw and processed malted sorghum sprout Department of Animal Production and Health, University of Agriculture, P.M.B. 2240, Abeokuta, Nigeria.

Gardner, C. D., Newell, K.A., Cherin, R and Haskell, W. (2001) The effect of soy protein with or without isoflavones relative to milk protein on plasma lipids in hypercholesterolemic postmenopausal women. American Journal of Clinical Nutrition, 73 (4): 728-735.

Gautam. M., Jain, A., Shakkarpude, J., Kushwah, M.S., and Jamre, B. (2017). Effect of germinated sorghum based diet at different levels on biochemical parameters in broilers. International Journal of Advance Biological Research, 7(1): 116-118.

Jain, A. (2008). Biochemical and haematological parameters in broilers. Indian Journal Animal Nutrition, 25(3):285-287.

Jamre, B. (2015). Evalution of diet containing germinated maize and multienzyme complex on growth and production performance of broilers. M.V.Sc \& A.H.Thesis. (Veterinary 
Physiology), Nanaji Deshmukh Veterinary Science University,Jabalpur (M.P).

Khan,M.A., Jain, Archana, Shakkarpude, J., Mehta, M.K., Kushwah M.S. \& Chouhan, Laxmi (2017) Effect of germinated maize and sorghum based diet at different levels on biochemical parameters in kadaknath. International Journal of Advance Biological Research, 7 (3): 552-555.

Kwari, I.D., Diarra, S.S., Igwebuike, J.U., Nkama, I., Issa, S., Hamaker, B.R., Hancock, J.D., Jauro, M., Seriki, O.A. and Murphy, T. (2012). Replacement value of low tannin Sorghum (Sorghum bicolor) for maize in Broiler chickens diet in the semi arid zone of Nigeria. International Journal Poultry Science, 11: 333-337.

Lee, D.W., Shin, J.H., Park, J.M., Song, J.C., Suh, H.J., Un-Jae Chang, U.J., Kang, C.W., and Kim, J.M., (2010). Growth Performance and Meat Quality of Broiler Chicks Fed Germinated and Fermented Soybeans. Korean Journal of Food Science Animal Resources, 30 (6): 938-945.

Manwar, S.J. and Mandal, A. B. (2009). Effect of reconstitution of sorghum with or without enzymes on production performance and immunocompetence in broiler chicken. Journal of the Science of Food and Agriculture, 89(6):998-1005.

Onifade, A. A., Obiyan, R. I., Onipede, E., Adejumo, D. O., Abu, O. A. and Babatunde, G. M. (1999). Assessment of the effect of supplement rabbit diets with a culture of Saccharomyces cerevisiae using performance, blood composition and clinical enzymes activities. Animal Feed Science Technology, 77:25-32.

Orban J. I., Adeola, O. and Stroshine, R.(1999) Microbial phytase in finisher diets of White
Pekin ducks: Effects on growth performance, plasma phosphorus concentration, and leg bone characteristics. Poultry Science. 78 : 366- 377.

Osman, A.H.E. (2009). Effect of multi enzyme supplementation and grain source on broiler performance and carcass quality. Animal Production Science and Technology, 70:2829.

Panda, B., and S. C. Mahapatra. (1989). Common breeds of poultry. Pages 6-18 in Poultry Production. ICAR, New Delhi, India.

Prajapati, R.S. (2010). Studies on phytase supplementation on growth performance and haemato-biochemical parameters in broilers. M.V.Sc \& A.H. Thesis (Veterinary Physiology), Rajmata Vijayaraje Scindia Krishi Vishwa Vidyalaya Gwalior.

Rao, G. V. and Thomas, P. C. (1984). The breed characteristics of Kadaknath breed of indigenous (Desi) chicken; Avian Research, 68: 55-57.

Shewry, Peter R., Johnathan A. Napier, and Arthur S. Tatham (1995) Seed Storage Proteins: Structures 'and Biosynthesis. The Plant Cell, 7: 945-956.

Singh, P.K. and Khatta, V.K. (2003). Effect of phytase supplementation on the performance of broiler chicken fed wheat based diets. Indian Journal Animal Nutrition, 20:57-62.

Snedecor, G.W. and Cochran,W.G. (1994). Statistical Methods, $8^{\text {th }}$ Edn., lowa state University press. Ames Iowa, $503 \mathrm{p}$.

Viveros, A., Brenes, A., Arija, I. and Centeno, C. (2002). Effects of microbial phytase supplementation on mineral utilization and serum enzyme activities in broiler chicks fed different levels of phosphorus. Poultry Science, 81: 1172-1183.

\section{How to cite this article:}

Akhelesh Kumar Karoriya, Archana Jain, M. K. Mehta, Chouhan, Laxmi, Kumar, Rakesh and Shakkarpude, J. 2019. Effect of Germinated Maize Based Diets on Biochemical Parameters in Kadaknath. Int.J.Curr.Microbiol.App.Sci. 8(09): 1072-1084.

doi: https://doi.org/10.20546/ijcmas.2019.809.125 